\title{
Sum-sets of small upper density
}

by

Guillaume Bordes (Bordeaux)

1. Introduction. Let $A \subseteq \mathbb{N}$ be an infinite set of non-negative integers. For $y>x>0$, we put

$$
A(x):=|A \cap[0 ; x]|, \quad A(x, y):=|A \cap[x ; y]| .
$$

We define the lower asymptotic density $\underline{d}(A)$ and upper asymptotic density $\bar{d}(A)$ by

$$
\underline{d}(A):=\liminf _{x \rightarrow \infty} \frac{A(x)}{x}, \quad \bar{d}(A):=\limsup _{x \rightarrow \infty} \frac{A(x)}{x} .
$$

Unless explicitly stated otherwise, we assume that

$$
0 \in A, \quad \operatorname{gcd}(A)=1 .
$$

We define the sum $X+Y$ of two sets $X, Y \subset \mathbb{R}$ by

$$
X+Y=\{x+y \mid x \in X, y \in Y\} .
$$

Inverse additive theory describes sets $A$ with "small" sum-set $A+A$. Say, one may ask about sets $A$ of positive lower or upper density with small quotient $\underline{d}(A+A) / \underline{d}(A)$ or $\bar{d}(A+A) / \bar{d}(A)$. For example, let $N \geq 3$ be an integer. Then for the set $A=\{0,1\}+N \mathbb{N}$ we have

$$
\underline{d}(A)=\bar{d}(A)=2 / N, \quad \underline{d}(A+A)=\bar{d}(A+A)=3 / N,
$$

so that the above-mentioned quotients are both equal to $3 / 2$. (As we shall see in a while, this is the minimal possible value under the assumption (1).)

Kneser $[7,4]$ gave a complete description of sets $A$ satisfying $\underline{d}(A+A)<$ $2 \underline{d}(A)$. In brief, he showed that $A$ should be "approximately" of the form $K+N \mathbb{N}$, where $N$ is a positive integer and $K$ is a set of residues $\bmod N$.

Among other things, Kneser's theorem implies that $\underline{d}(A+A) \geq \frac{3}{2} \underline{d}(A)$ when $A$ satisfies (1), and the equality $\underline{d}(A+A)=\frac{3}{2} \underline{d}(A)$ is possible only with $|K|=2$.

2000 Mathematics Subject Classification: Primary 11P70. 
Extending Kneser's results to upper density seems to be a rather difficult problem. The following example, due to Jin [5], shows that this time one cannot get away with sets of the type $K+N \mathbb{N}$.

EXAMPLE 1.1. Let $\alpha$ be a real number satisfying $0<\alpha<1 / 2$. Let $\left(T_{n}\right)_{n \geq 1}$ be an increasing sequence of positive integers such that $\lim T_{n+1} / T_{n}=\infty$. Then the set

$$
A=\mathbb{N} \cap \bigcup_{n=1}^{\infty}\left[\left\lceil(1-\alpha) T_{n}\right\rceil, T_{n}\right]
$$

satisfies $\bar{d}(A)=\alpha$ and $\bar{d}(A+A)=\frac{3}{2} \alpha$.

In what follows, we use the notation

$$
\alpha=\bar{d}(A), \quad \gamma=\bar{d}(A+A) .
$$

We assume that $0<\alpha \leq 1 / 2$ and we put $\sigma=\gamma / \alpha$.

It is not difficult to show that $\sigma \geq 3 / 2$ (see Lemma 2.1 below), but the structure of sets $A$ with $\sigma=3 / 2$ was only recently determined by Jin [6]. He proved that a set $A$ with $\sigma=3 / 2$ is "similar" either to $K+N \mathbb{N}$ with $|K|=2$, or to the set from Example 1.1. For $\sigma>3 / 2$ the problem is open.

In the present article we determine the structure of sets $A$ with $3 / 2 \leq \sigma$ $<5 / 3$ subject to the additional assumption $\alpha<\alpha_{0}$, where $\alpha_{0}$ is a small absolute constant. For $\sigma=3 / 2$ our result is covered by that of Jin, but for $3 / 2<\sigma<5 / 3$ our result is new.

Now, we can formulate the main result of this article.

THEOREM 1.2. There exists a positive absolute constant $\alpha_{0}$ such that the following holds. Let $A$ be a set of non-negative integers such that $0 \in A$ and $\operatorname{gcd}(A)=1$. Put $\alpha=\bar{d}(A)$ and $\gamma=\bar{d}(A+A)$. Assume that $0<\alpha=\bar{d}(A)$ $\leq \alpha_{0}$ and that

$$
\gamma=\sigma \alpha, \quad \text { where } 3 / 2 \leq \sigma<5 / 3 .
$$

Then we have one of the following cases:

1. Non-archimedean case: there exist two positive integers $N$ and $t$ with $\operatorname{gcd}(N, t)=1$ such that $A \subseteq\{0, t\}+N \mathbb{N}$, and

$$
\alpha \geq \frac{6}{(4 \sigma-3) N} .
$$

2. Archimedean case: there exists an increasing sequence $\left(y_{j}\right)_{j \geq 1}$ of integers with

$$
\lim _{j \rightarrow \infty} \frac{A\left(y_{j}\right)}{y_{j}}=\alpha,
$$

and two sequences $\left(b_{j}\right)_{j \geq 1}$ and $\left(t_{j}\right)_{j \geq 1}$ with $0 \leq b_{j} \leq t_{j} \leq y_{j}$ such that, 
if we define

$$
\lambda_{j}:=\frac{b_{j}}{y_{j}-t_{j}}, \quad r_{j}:=\frac{A\left(t_{j}, y_{j}\right)}{y_{j}-t_{j}+1},
$$

then $A\left(b_{j}, t_{j}\right)=0$ for all $j \geq 1$ and

$$
\lim _{j \rightarrow \infty} \lambda_{j}=\lambda, \quad \lim _{j \rightarrow \infty} r_{j}=r
$$

with

$$
\lambda \leq \frac{2 \sigma-3}{2 \sigma-2}\left(\frac{1}{2 \sigma-2}-\alpha\right)^{-1}, \quad r \geq\left(\frac{1}{2 \sigma-2}+\lambda\left(\frac{1}{2 \sigma-2}-\alpha\right)\right) .
$$

EXAMPLE 1.3. We cannot extend Theorem 1.2 to the case $\bar{d}(A+A)=$ $\frac{5}{3} \bar{d}(A)$. It suffices to consider the set $A:=N \mathbb{N} \cup(1+2 N \mathbb{N})$ which satisfies this condition and for which $\alpha=3 / 2 N$. Putting $\sigma=5 / 3$ in Theorem 1.2 would give $\alpha \geq 18 / 11 N>3 / 2 N$.

The following example proves that the lower bound obtained in the nonarchimedean case of Theorem 1.2 cannot be refined.

EXAmple 1.4. Fix $3 / 2 \leq \sigma<5 / 3$. Let $\left(T_{n}\right)_{n \geq 1}$ be an increasing sequence of positive integers such that $\lim _{n \rightarrow \infty} T_{n+1} / T_{n}=\infty$ and set

$$
E:=\bigcup_{n=1}^{\infty}\left[\left\lceil\left(1-\alpha^{\prime}\right) T_{n}\right\rceil ; T_{n}\right]
$$

where $\alpha^{\prime}=3 /(4 \sigma-3)$. Let $N$ be a sufficiently large positive integer and

$$
A:=N E \cup(1+N E) .
$$

We can verify that

$$
\alpha=\frac{6}{(4 \sigma-3) N}<\alpha_{0}, \quad \gamma=3 \frac{1+\alpha^{\prime}}{2 N}=\frac{6 \sigma}{(4 \sigma-3) N} .
$$

2. General results in additive number theory. Before proving the main theorem, let us show why $3 / 2$ is a lower bound for the quotient $\sigma$.

Lemma 2.1. Let $A$ be a set of non-negative integers. Suppose that $0 \in A$ and $\operatorname{gcd}(A)=1$. Then $\gamma \geq \frac{3}{2} \alpha$.

We can easily deduce the lemma from the following

Theorem 2.2 ([9, p. 23]). Let $k \geq 3$ be an integer. Let $A=\left\{a_{0}, a_{1}, \ldots\right.$ $\left.\ldots, a_{k-1}\right\}$ be a set of non-negative integers such that

$$
0=a_{0}<a_{1}<\cdots<a_{k-1}, \quad \operatorname{gcd}(A)=1 .
$$

If $a_{k-1} \geq 2 k-3$, then $|A+A| \geq 3 k-3$. 
Proof of Lemma 2.1. Since $\bar{d}(A)=\alpha$, there exists an increasing sequence $\left(y_{j}\right)_{j \geq 1}$ of integers such that, for all $\varepsilon>0$, if we define $A_{j}:=A \cap\left[0 ; y_{j}\right]$ and assume $j$ sufficiently large, we have

$$
\alpha-\varepsilon<\left|A_{j}\right| / y_{j}<\alpha+\varepsilon, \quad \operatorname{gcd}\left(A_{j}\right)=1 .
$$

In what follows, we will assume that $y_{j} \in A_{j}$.

Under the hypothesis $\alpha<1 / 2$, we can see that $A_{j}$ satisfies the hypothesis of Theorem 2.2. Thus,

$$
\left|A_{j}+A_{j}\right| \geq 3\left|A_{j}\right|-3
$$

and therefore,

$$
\frac{(A+A)\left(2 y_{j}\right)}{2 y_{j}} \geq \frac{\left|A_{j}+A_{j}\right|}{2 y_{j}} \geq \frac{3}{2} \frac{\left|A_{j}\right|}{y_{j}}-\frac{3}{2 y_{j}} \geq \frac{3}{2} \alpha-2 \varepsilon .
$$

This yields $\bar{d}(A+A) \geq \frac{3}{2} \alpha$ and concludes the proof.

In the rest of this section, we give some general results in additive number theory, to be used in the next section.

Let $A$ be a finite set of integers. It is easy to see that $|A+A| \geq 2|A|-1$, and $|A+A|=2|A|-1$ if and only if $A$ is an arithmetical progression.

Freiman $[9$, p. 21] generalized this fact.

Theorem 2.3 (Freiman). Let $A$ be a finite set of non-negative integers such that $|A| \geq 3$ and $\min (A)=0$. Denote by $a_{k}$ the greatest element of $A$. If

$$
a_{k} \leq 2|A|-3
$$

then

$$
|2 A| \geq|A|+a_{k} .
$$

This result has been generalized to distinct sets by V. F. Lev and P. Y. Smeliansky in [8] and was improved by Y. V. Stanchescu in [10]. We will use the following version:

Theorem 2.4 (Lev, Smeliansky). Let $A$ and $B$ be two finite sets of nonnegative integers such that $0 \in A \cap B$. Denote by $l(A):=\max (A)-\min (A)$ the length of $A$ and by $h(A):=l(A)-|A|+1$ the number of holes in $A$. If

$$
\max (l(A), l(B)) \leq|A|+|B|-3
$$

then

$$
|A+B| \geq(|A|+|B|-1)+\max (h(A), h(B)) .
$$

Now, let us introduce some notions taken from [2].

Definition 2.5. Let $A$ and $B$ be two abelian groups and $K \subset A, L \subset B$. A map $\varphi: K \rightarrow L$ is said to be a Freiman homomorphism or an $F_{2}$ homomorphism if, for all $\left(x, y, x^{\prime}, y^{\prime}\right) \in K^{4}$, we have

$$
x+y=x^{\prime}+y^{\prime} \Rightarrow \varphi(x)+\varphi(y)=\varphi\left(x^{\prime}\right)+\varphi\left(y^{\prime}\right) .
$$


Such a $\varphi$ is said to be an $F_{2}$-isomorphism if it is invertible and if $\varphi^{-1}$ is also an $F_{2}$-homomorphism.

REMARK 2.6. In what follows, we will use some affine maps in $\mathbb{Z}^{2}$ which are clearly $F_{2}$-isomorphisms.

The following proposition is clear:

Proposition 2.7. An $F_{2}$-isomorphism $\varphi: K \rightarrow L$ induces a bijective map $K+K \rightarrow L+L$.

REMARK 2.8. Similarly, for any positive integer $i$ we can define the notion of $F_{i}$-homomorphism. We say that $\varphi: K \rightarrow L$ is an $F_{i}$-homomorphism if for all $\left(x_{1}, \ldots, x_{i}, x_{1}^{\prime}, \ldots, x_{i}^{\prime}\right) \in K^{2 i}$,

$x_{1}+\cdots+x_{i}=x_{1}^{\prime}+\cdots+x_{i}^{\prime} \Rightarrow \varphi\left(x_{1}\right)+\cdots+\varphi\left(x_{i}\right)=\varphi\left(x_{1}^{\prime}\right)+\cdots+\varphi\left(x_{i}^{\prime}\right)$.

Clearly, an $F_{i}$-homomorphism is an $F_{2}$-homomorphism for any $i \geq 2$.

Definition 2.9. A subset $P$ of an abelian group is called a generalized arithmetical progression of dimension $m$ if it can be written as

$$
\begin{aligned}
P & =P\left(x_{0} ; x_{1}, \ldots, x_{m} ; b_{1}, \ldots, b_{m}\right) \\
& =\left\{x_{0}+\beta_{1} x_{1}+\cdots+\beta_{m} x_{m}: \beta_{i}=0, \ldots, b_{i}-1\right\}
\end{aligned}
$$

where $x_{0}, \ldots, x_{m}$ are elements of the group and $b_{1}, \ldots, b_{m}$ are positive integers.

We say that $P$ is an $F_{2}$-progression if the map

$$
\begin{aligned}
\theta:\left\{0, \ldots, b_{1}-1\right\} \times \cdots \times\left\{0, \ldots, b_{m}-1\right\} & \subset \mathbb{Z}^{m} \rightarrow P, \\
\left(\beta_{1}, \ldots, \beta_{m}\right) & \mapsto x_{0}+\beta_{1} x_{1}+\cdots+\beta_{m} x_{m},
\end{aligned}
$$

is an $F_{2}$-isomorphism.

We will heavily use the following fundamental theorem due to G. Freiman whose proof can be found in [2] and whose version below is taken from [1]:

THEOREM 2.10 (Freiman). Let $\sigma$ be a positive real number, and $A$ a finite set of non-negative integers such that $0 \in A$ and $|A|>k(\sigma)$ where $k=k(\sigma)$ is a fixed constant depending only on $\sigma$. If

$$
|A+A| \leq \sigma|A|
$$

then $A$ is a subset of an $F_{2}$-progression

$$
P=P\left(0 ; x_{1}, \ldots, x_{m} ; b_{1}, \ldots, b_{m}\right)
$$

of dimension $m \leq\lfloor\sigma-1\rfloor$ and whose length is bounded from above: $|P| \leq$ $C_{1}(\sigma)|A|$.

Furthermore, if $b_{1} \leq \cdots \leq b_{m}$, then

$$
i>\left\lfloor\log _{2} \sigma\right\rfloor \Rightarrow b_{i} \leq C_{2}(\sigma) .
$$

Here $C_{1}(\sigma)$ and $C_{2}(\sigma)$ are constants depending only on $\sigma$. 
Our strategy of proof is simple. First, we are going to transform the infinite problem into a finite one. Then we will use Theorem 2.10 to obtain the structure of finite sets. Finally, we will come back to the set $A$ using asymptotic arguments.

In the following, Theorem 2.10 will be used with $\sigma<4$ so that it will give rise to $F_{2}$-progressions of dimension at most 2 . Then, in view of Definition 2.9, it will be natural to use results concerning addition of sets in $\mathbb{Z}^{2}$, particularly the following one whose proof can be found in [3, p. 28]:

Theorem 2.11 (Freiman). Let $A \subset \mathbb{Z}^{2}$ be a set of at least twelve elements not on the same line. Assume that

$$
|A+A|<\frac{10}{3}|A|-5 .
$$

Then $A$ is contained in a set $F_{2}$-isomorphic to

$$
A^{0}=\left\{(0,0),(0,1), \ldots,\left(0, l_{1}-1\right)\right\} \cup\left\{(1,0),(1,1), \ldots,\left(1, l_{2}-1\right)\right\}
$$

with $l_{1}, l_{2} \geq 1$ and $l_{1}+l_{2}=|A+A|-2|A|+3$.

3. Proof of the main theorem. With a view to use the theorems of the previous section, let us transform our problem into a problem on finite sets.

Let $\varepsilon>0$. We can choose $y_{1} \in \mathbb{N}$ sufficiently large and a strictly increasing sequence $\left(y_{j}\right)_{j \geq 1}$ of positive integers such that for all $j$,

$$
(A+A)\left(2 y_{j}\right) \leq(\gamma+\varepsilon) \cdot 2 y_{j}, \quad(\alpha-\varepsilon) y_{j} \leq A\left(y_{j}\right) \leq(\alpha+\varepsilon) y_{j} .
$$

We will use the notation

$$
A_{j}:=\left\{a \in A: a \leq y_{j}\right\} .
$$

In what follows, all the notations will depend on the sequence $\left(y_{j}\right)_{j \geq 1}$. Every change of the sequence will naturally change the sets $A_{j}$ and all related objects. We will denote by $O(\varepsilon)$ any positive function of $\varepsilon$ bounded above by $C \varepsilon$ where $C$ is a constant only depending on the set $A$.

Now, we are able to determine the structure of the sets $A_{j}$. We have

$$
\begin{aligned}
\frac{\left|A_{j}+A_{j}\right|}{\left|A_{j}\right|} & =\frac{\left|A_{j}+A_{j}\right|}{2 y_{j}} \cdot 2 \cdot \frac{y_{j}}{\left|A_{j}\right|} \\
& \leq \frac{(A+A)\left(2 y_{j}\right)}{2 y_{j}} \cdot 2 \cdot \frac{y_{j}}{\left|A_{j}\right|} \\
& \leq 2 \cdot \frac{\gamma+\varepsilon}{\alpha-\varepsilon} \leq 2 \sigma+\varepsilon^{\prime}<4,
\end{aligned}
$$

where $\varepsilon^{\prime}=O(\varepsilon)$. Thus, for $\varepsilon$ sufficiently small, we can apply the fundamental Theorem 2.10 of Freiman to the sets $A_{j}$. By a simple calculation, we obtain $m \leq 2$ and $b_{2} \leq C_{2}$. First, we are going to exclude the case where $A_{j}$ is a 
subset of an arithmetical progression of dimension $m=1$ for infinitely many values of $j$.

Suppose this is the case. Then, for $j$ sufficiently large, $A_{j} \subseteq P_{j}$ where $P_{j}$ is an arithmetical progression of difference $1($ for $\operatorname{gcd}(A)=1)$ and first term 0 . We can assume it has minimal length. Then, by Theorem 2.10 and since $\left\{0, y_{j}\right\} \subseteq P_{j}$, we have

$$
\begin{aligned}
& \left|P_{j}\right| \geq y_{j}, \\
& \left|P_{j}\right| \leq C_{1}\left|A_{j}\right| .
\end{aligned}
$$

Now we combine (4) and (5) to find a lower bound for $\alpha$ :

$$
\begin{aligned}
\alpha & \geq \frac{1}{\sigma} \gamma \geq \frac{1}{\sigma}\left(\frac{\left|A_{j}+A_{j}\right|}{2 y_{j}}-\varepsilon\right) \geq \frac{1}{\sigma}\left(\frac{2\left|A_{j}\right|-1}{2 y_{j}}-\varepsilon\right) \\
& \geq \frac{1}{\sigma}\left(\frac{1}{2 y_{j}}\left(\frac{2 y_{j}}{C_{1}}-1\right)-\varepsilon\right) \geq \alpha_{0},
\end{aligned}
$$

for an absolute constant $\alpha_{0}$ (remember that $\varepsilon$ can be chosen sufficiently small). Thus, we can exclude this case under hypothesis $\alpha<\alpha_{0}$ of Theorem 1.2 .

Remark 3.1. The value of $C_{1}$ (one can find an estimate in [2]) implies a very small value for the bound $\alpha_{0}$. What happens for $\alpha>\alpha_{0}$ is an open question.

Thus, for infinitely many integers $j$, the set $A_{j}$ is a subset of an arithmetical progression of dimension $m=2$. By extracting a subsequence, we can assume that this is the case for all $A_{j}$. Then, for all $j \geq 1$, there is an $F_{2}$-isomorphism $\theta_{j}$ between a subset of $\mathbb{Z}^{2}$ and $A_{j}$ (see Definition 2.9). By Proposition 2.7 , the sets $\theta_{j}^{-1}\left(A_{j}\right)$ satisfy the inequality

$$
\left|\theta_{j}^{-1}\left(A_{j}\right)+\theta_{j}^{-1}\left(A_{j}\right)\right| \leq\left(2 \sigma+\varepsilon^{\prime}\right)\left|\theta_{j}^{-1}\left(A_{j}\right)\right| .
$$

At this point, using the assumption $\sigma<5 / 3$, we can apply Theorem 2.11 to $\theta_{j}^{-1}\left(A_{j}\right)$. Composing isomorphisms, we see that, for all $j \geq 1$, there exists an $F_{2}$-isomorphism $\varphi_{j}: \mathbb{Z}^{2} \rightarrow \mathbb{N}$ such that $A_{j} \subseteq \varphi_{j}\left(A_{j}^{0}\right)$ where $A_{j}^{0}=$ $\left\{(0,0),(0,1), \ldots,\left(0, l_{1, j}-1\right)\right\} \cup\left\{(1,0),(1,1), \ldots,\left(1, l_{2, j}-1\right)\right\}$. Combining, if necessary, those isomorphisms with suitable affine maps, we can assume that $\varphi_{j}((0,0)) \in A_{j}$ and $\varphi_{j}((1,0)) \in A_{j}$. Furthermore, we have $l_{1, j}+l_{2, j}=$ $\left|A_{j}+A_{j}\right|-2\left|A_{j}\right|+3$.

Notice that the number of elements of $\varphi^{-1}\left(A_{j}\right)$ in each line cannot be bounded, since otherwise, for all $\varepsilon>0$, we could obtain $\bar{d}(A+A)>$ $(2-\varepsilon) \bar{d}(A)$ by considering the sequence $(A+A)\left(y_{j}\right) / y_{j}$.

We set $d_{1, j}:=\varphi_{j}((1,0))-\varphi_{j}((0,0))$ and $d_{2, j}:=\varphi_{j}((0,1))-\varphi_{j}((0,0))$. 
Then we can give the explicit $F_{2}$-isomorphism

$$
\varphi_{j}: \mathbb{Z} \times\{0,1\} \rightarrow \mathbb{N}, \quad(x, y) \mapsto a_{j}+x d_{1, j}+y d_{2, j},
$$

where $a_{j}=\varphi_{j}((0,0))$.

Since $A \subseteq \mathbb{N}$, the number $d_{1, j}$ has to be positive for infinitely many values of $j$ which we again extract. We can also assume, by switching the lines if necessary, that the differences $d_{2, j}$ are positive.

Lemma 3.2. The sequence $\left(d_{1, j}\right)_{j \geq 1}$ is bounded.

Proof. Assume the contrary. Then there exists an index $j$ such that $A\left(d_{1, j}\right)>3$ and, consequently, there exist distinct $a, b \in A \cap\left[0 ; d_{1, j}\right]$ such that $\varphi_{j}^{-1}(a)$ and $\varphi_{j}^{-1}(b)$ lie on the same line. We deduce from (7) that $|b-a|=$ $k d_{1, j}$ where $k$ is a positive integer. This is impossible since $|b-a|<d_{1, j}$.

Since the sequence $\left(d_{1, j}\right)_{j \geq 1}$ is bounded, there exists a positive integer $N$ such that $d_{1, j}=N$ for infinitely many $j$. We choose the largest $N$ with this property, and, again extracting a subsequence, we assume that $d_{1, j}=N$ for all $j$.

3.1. The non-archimedean case. In this case, we assume $N>1$. We show that the sequence $\left(d_{2, j}\right)_{j \geq 1}$ can then be supposed to be constant.

Lemma 3.3. There exist a positive integer $t$ and a sequence $\left(y_{j}\right)_{j \geq 1}$ such that $d_{2, j}=t$ for all $j \geq 1$.

Proof. Each $A_{j}$ is included in two residue classes $\bmod N$. Since those sets satisfy $A_{j} \subseteq A_{k}$ for $j<k$, the whole set $A$ is included in two residue classes. If we denote by $t$ the smallest term of the part of $A$ not congruent to $0 \bmod N$, we can choose, for each $j \geq 1$, the isomorphism $\varphi_{j}$ such that $\varphi_{j}((0,0))=0$ and $\varphi_{j}((1,0))=t$.

Hence, we can assume that $d_{2, j}=t$ for all $j \geq 1$ and we can exhibit an $F_{2}$-isomorphism $\varphi$ between $\mathbb{Z}^{2}$ and $\mathbb{N}$ such that $\varphi_{\mid A_{j}}=\varphi_{j}$ :

$$
\varphi: \mathbb{Z} \times\{0,1\} \rightarrow \mathbb{N}, \quad(x, y) \mapsto x N+y t .
$$

By hypothesis (1), we must have $\operatorname{gcd}(t, N)=1$ and $A$ is included in two residue classes $\bmod N$ which we denote by $B$ and $C$ :

$$
B=\{a \in A: a \equiv 0 \bmod N\}, \quad C=\{a \in A: a \equiv t \bmod N\} .
$$

We define $B_{j}:=B\left(y_{j}\right)$ and $C_{j}:=C\left(y_{j}\right)$ and we assume, choosing $y_{1}$ sufficiently large, that those sets are non-empty. We define $t_{0}:=\min (C)$, $b_{j}:=\max \left(B_{j}\right)$ and $c_{j}:=\max \left(C_{j}\right)$. We may assume that $b_{j}=y_{j}$, replacing if necessary $A$ by $A-t_{0}$ and extracting a subsequence of $\left(y_{j}\right)_{j \geq 1}$. 
Lemma 3.4. There exists a sequence $\left(y_{j}\right)_{j \geq 1}$ such that, for all $\varepsilon>0$ and for $j$ sufficiently large,

$$
\left|A_{j}\right| \geq \frac{1}{(2 \sigma-2+\varepsilon) N}\left(b_{j}+c_{j}\right) .
$$

Proof. Remember that $t_{0}$ is the smallest element of $A$ not divisible by $N$. We define $S_{j}:=b_{j}+c_{j}-t_{0}+2$.

Let $\varepsilon>0$. We have, using Theorem 2.11,

$$
\frac{S_{j}}{N} \leq\left|A_{j}+A_{j}\right|-2\left|A_{j}\right|+3 \leq\left(2 \sigma-2+\varepsilon^{\prime}\right)\left|A_{j}\right|+3 \leq\left(2 \sigma-2+\varepsilon^{\prime \prime}\right)\left|A_{j}\right|,
$$

where $\varepsilon^{\prime}=O(\varepsilon)$ and $\varepsilon^{\prime \prime}=O(\varepsilon)$. It suffices to choose $j$ sufficiently large to obtain the result.

Below, $\left(y_{j}\right)_{j \geq 1}$ is a sequence of integers as in the last lemma.

Now, we are going to refine the last results. We define

$$
X_{j}:=\frac{c_{j}}{b_{j}}, \quad \lambda_{j}:=\frac{N\left|A_{j}\right|}{b_{j}+c_{j}} .
$$

Lemma 3.5. There exists a sequence $\left(y_{j}\right)_{j \geq 1}$ such that $\lim _{j \rightarrow \infty} X_{j}=1$.

Proof. We will only use the definition of the upper asymptotic density of $A$. Given $\varepsilon>0$, for all sufficiently large $j$ we have

$$
\frac{A\left(c_{j}\right)}{c_{j}} \leq \frac{A\left(b_{j}\right)}{b_{j}}+\varepsilon
$$

Furthermore,

$$
A\left(c_{j}\right) \geq A\left(b_{j}\right)-\frac{b_{j}-c_{j}}{N} .
$$

Putting together the last two relations, we obtain

$$
N \varepsilon+\frac{\lambda_{j}\left(b_{j}+c_{j}\right)}{b_{j}} \geq \frac{\lambda_{j}\left(b_{j}+c_{j}\right)}{c_{j}}-\frac{b_{j}}{c_{j}}+1 .
$$

This yields the following polynomial inequality:

$$
\lambda_{j} X_{j}^{2}-(1-N \varepsilon) X_{j}-\left(\lambda_{j}-1\right) \geq 0 .
$$

It remains to determine the discriminant and the roots. We obtain

$$
\Delta=\left(2 \lambda_{j}-1\right)^{2}+\varepsilon\left(N^{2} \varepsilon-2 N\right) .
$$

Thus, using Lemma 3.4 to bound $\lambda_{j}$ from below, we see that the roots $X_{j}^{\prime}<X_{j}^{\prime \prime}$ satisfy

$$
\begin{aligned}
& X_{j}^{\prime}=\frac{1}{2 \lambda_{j}}(1-N \varepsilon-\sqrt{\Delta})=\frac{1}{\lambda_{j}}-1+O(\varepsilon), \\
& X_{j}^{\prime \prime}=\frac{1}{2 \lambda_{j}}(1-N \varepsilon+\sqrt{\Delta})=1-O(\varepsilon) .
\end{aligned}
$$


Clearly, $X_{j}<1 / \lambda_{j}-1+O(\varepsilon)$ is impossible, since the lower bound on $\lambda_{j}$ obtained in Lemma 3.4 would imply

$$
X_{j} \leq 1 / \lambda_{j}-1+O(\varepsilon) \leq 2 \sigma-3+O(\varepsilon)<1 / 3
$$

for $\varepsilon$ sufficiently small, and hence

$$
\frac{(A+A)\left(b_{j}+t_{0}\right)}{b_{j}+t_{0}} \geq \frac{\left|B_{j}\right|+\left|B_{j}\right|+\left|C_{j}+C_{j}\right|}{b_{j}+t_{0}} \geq 2 \alpha-O(\varepsilon),
$$

which contradicts the main hypothesis of Theorem 1.2. Thus, $X_{j} \geq 1-O(\varepsilon)$, which is the conclusion of the lemma.

Now we combine the results of the last two lemmas and apply Theorems 2.3 and 2.4 to the sets

$$
B_{j}^{\prime}:=\frac{1}{N} B_{j}, \quad C_{j}^{\prime}:=\frac{1}{N}\left(C_{j}-t\right) .
$$

We have

$$
\left|A_{j}\right| \geq \frac{1}{(2 \sigma-2-\varepsilon) N}\left(b_{j}+c_{j}\right) .
$$

We notice that, for $\varepsilon$ sufficiently small, since $\sigma<5 / 3$,

$$
\frac{1}{2 \sigma-2-\varepsilon}>\frac{3}{4}
$$

Fix $\delta>0$ such that

$$
\left|A_{j}\right| \geq\left(\frac{3}{4}+\delta\right) \frac{b_{j}+c_{j}}{N}
$$

Using Lemma 3.5, we obtain

$$
\left|A_{j}\right| \geq\left(\frac{3}{4}+\delta\right) \frac{\left(2-\varepsilon^{\prime}\right) y_{j}}{N}
$$

for $\varepsilon^{\prime}$ arbitrarily small. Therefore, there exists a positive constant $\delta^{\prime}$ such that, for $j$ sufficiently large,

$$
\left|A_{j}\right| \geq\left(\frac{3}{2}+\delta^{\prime}\right) \frac{y_{j}}{N}
$$

It follows that

$$
\left|B_{j}^{\prime}\right|=\left|B_{j}\right|=\left|A_{j}\right|-\left|C_{j}\right| \geq\left|A_{j}\right|-\frac{y_{j}}{N} \geq\left(\frac{1}{2}+\delta^{\prime}\right) \frac{y_{j}}{N} \geq\left(\frac{1}{2}+\delta^{\prime}\right) \max \left(B_{j}^{\prime}\right) .
$$

Thus we can apply Theorem 2.3 to $B_{j}^{\prime}$. We can do the same for $C_{j}^{\prime}$. Moreover, we have

$$
\left|B_{j}^{\prime}\right|+\left|C_{j}^{\prime}\right|=\left|A_{j}\right| \geq\left(\frac{3}{2}+\delta^{\prime}\right) \frac{y_{j}}{N},
$$

so we can also apply Theorem 2.4. 
For $j$ sufficiently large, we then have

$$
\begin{aligned}
\left|A_{j}+A_{j}\right| & =\left|B_{j}+B_{j}\right|+\left|B_{j}+C_{j}\right|+\left|C_{j}+C_{j}\right| \\
& =\left|B_{j}^{\prime}+B_{j}^{\prime}\right|+\left|B_{j}^{\prime}+C_{j}^{\prime}\right|+\left|C_{j}^{\prime}+C_{j}^{\prime}\right| \\
& \geq\left|B_{j}^{\prime}\right|+\frac{y_{j}}{N}+\left(1-\varepsilon^{\prime}\right) \frac{y_{j}}{N}+\left|B_{j}^{\prime}\right|+\left(1-\varepsilon^{\prime}\right) \frac{y_{j}}{N}+\left|C_{j}^{\prime}\right| \\
& =2\left|B_{j}\right|+\left|C_{j}\right|+\left(3-2 \varepsilon^{\prime}\right) \frac{y_{j}}{N},
\end{aligned}
$$

assuming, without loss of generality, that $\left|B_{j}\right| \geq\left|C_{j}\right|$. Here, $\varepsilon^{\prime}$ is arbitrarily small, by Lemma 3.5.

Now, we also have, for $j$ sufficiently large,

$$
\left|A_{j}+A_{j}\right| \leq\left(2 \sigma+\varepsilon^{\prime}\right)\left|A_{j}\right|
$$

Since $\left|B_{j}\right| \geq\left|C_{j}\right|$, inequality (9) implies that

$$
\left|A_{j}+A_{j}\right| \geq \frac{3}{2}\left|B_{j}\right|+\frac{3}{2}\left|C_{j}\right|+\left(3-2 \varepsilon^{\prime}\right) \frac{y_{j}}{N}=\frac{3}{2}\left|A_{j}\right|+\left(3-2 \varepsilon^{\prime}\right) \frac{y_{j}}{N} .
$$

Combining this with (10), we obtain

$$
\left|A_{j}\right| \geq \frac{6-4 \varepsilon^{\prime}}{4 \sigma-3+2 \varepsilon^{\prime}} \frac{y_{j}}{N} .
$$

Now, dividing by $y_{j}$ and sending $j$ to infinity, we obtain

$$
\alpha \geq \frac{6-4 \varepsilon^{\prime}}{4 \sigma-3+2 \varepsilon^{\prime}} \frac{1}{N} .
$$

Since $\varepsilon^{\prime}$ is arbitrary, this proves the required inequality of Theorem 1.2:

$$
\bar{d}(A) \geq \frac{6}{(4 \sigma-3) N} .
$$

3.2. The archimedean case. Now we assume that $N=1$. We show that, in this case, the sequence $\left(d_{2, j}\right)_{j \geq 1}$ cannot be bounded. Suppose the contrary; then we could extract a subsequence of $\left(y_{j}\right)_{j \geq 1}$ such that $d_{2, j}=t$ for all $j$ and act as in the non-archimedean case, i.e. find a common isomorphism between every $A_{j}$ and a part of two lines of $\mathbb{Z}^{2}$. This isomorphism could be written

$$
\varphi: \mathbb{Z} \times\{0,1\} \rightarrow \mathbb{N}, \quad(x, y) \mapsto x+t y .
$$

This is impossible because, for $j$ sufficiently large, we would have an element of $A \cap \varphi(\{y=0\})$ greater than $t$ (remember that there are infinitely many elements of $\varphi^{-1}(A)$ on each line) so that $t$ would have two inverse images under $\varphi$ (one on each line), which contradicts the definition of an $F_{2}$-isomorphism.

Therefore, we can choose $\left(y_{j}\right)_{j \geq 1}$ and consequently $t_{j}:=d_{2, j}$ such that $t_{j}$ is a strictly increasing sequence. Thus, as in the non-archimedean case, 
we can have $\varphi_{j}((0,0))=0, \varphi_{j}((1,0))=1, \varphi_{j}((1,0))=t_{j}$ and

$$
\varphi_{j}: \mathbb{Z} \times\{0,1\} \rightarrow \mathbb{N}, \quad(x, y) \mapsto x+y t_{j} .
$$

We shall apply Theorem 2.11 to the sets $A_{j}$. Then, we can include $A_{j}$ in a set $A_{j}^{0}$ which is the union of two arithmetical progressions $B_{j}^{0}$ and $C_{j}^{0}$ (of difference $N=1$ here). We denote as usual by $b_{j}:=l_{2, j}=\left|B_{j}^{0}\right|$ and $c_{j}:=l_{2, j}=\left|C_{j}^{0}\right|$ the respective lengths, where $0 \in B_{j}^{0}$ and $y_{j} \in C_{j}^{0}$. Indeed, those two elements cannot be in the same progression: in this case, $A$ would be in an arithmetical progression of dimension 1 , say $B_{j}^{0}$. This case, which is the single line case, is already excluded by $\alpha<\alpha_{0}$. Those lengths being supposed minimal, we have $y_{j}-t_{j}=l_{2, j}$ and $\max \left(B_{j}^{0}\right)=b_{j}$.

Lemma 3.6. There exists a sequence $\left(y_{j}\right)_{j \geq 1}$ such that, for all $\varepsilon>0$, there exists $j_{0} \geq 1$ such that for all $j \geq j_{0}$,

$$
\left|A_{j}\right| \geq\left(\frac{1}{2 \sigma-2}-\varepsilon\right)\left(l_{1, j}+l_{2, j}\right) .
$$

Proof. It suffices to apply Theorem 2.11 for $j$ sufficiently large:

$$
\begin{aligned}
l_{1, j}+l_{2, j} & \leq\left|A_{j}+A_{j}\right|-2\left|A_{j}\right|+3 \leq\left(2 \sigma-2+\varepsilon^{\prime}\right)\left|A_{j}\right|+3 \\
& \leq\left(2 \sigma-2+\varepsilon^{\prime \prime}\right)\left|A_{j}\right|,
\end{aligned}
$$

where $\varepsilon^{\prime}$ is arbitrarily small and $\varepsilon^{\prime \prime}=O\left(\varepsilon^{\prime}\right)$.

From now on, $\left(y_{j}\right)_{j \geq 1}$ is a sequence of integers as in the last lemma.

If $b_{j} \geq t_{j}$, then $l_{1, j}+l_{2, j} \geq y_{j}$ and, by Lemma 3.6 and the range of values of $\sigma$, we have $\left|A_{j}\right| \geq \frac{3}{4} y_{j}$, which is incompatible with $\alpha<1 / 2$. Therefore, $b_{j}<t_{j}$, and thus

$$
A\left(b_{j}, t_{j}\right)=0 .
$$

Now we define $B_{j}:=A \cap\left[0 ; b_{j}\right]$ and $C_{j}:=A \cap\left[t_{j} ; y_{j}\right]$ with $b_{j}<t_{j}$.

The quotient $X_{j}:=\left|B_{j}\right| / b_{j}$ cannot be too large, otherwise we would obtain, considering the sets $A\left(b_{j}\right)$, a too large value for $\alpha$. Clearly, we have

$$
X:=\limsup _{j \rightarrow \infty} X_{j} \leq \alpha .
$$

Let us show in which sense $b_{j}$ is necessarily small compared with $l_{2, j}$.

Lemma 3.7. Define $\lambda_{j}:=b_{j} / l_{2, j}$. Then

$$
\lambda:=\limsup _{j \rightarrow \infty} \lambda_{j} \leq \frac{2 \sigma-3}{2 \sigma-2}\left(\frac{1}{2 \sigma-2}-X\right)^{-1} .
$$

Proof. We use Lemma 3.6, noting that

$$
\left|A_{j}\right|=\left|B_{j}\right|+\left|C_{j}\right|=X_{j} \lambda_{j} l_{2, j}+\left|C_{j}\right| .
$$


For all $\varepsilon>0$, and $j$ sufficiently large, we obtain

$$
X_{j} \lambda_{j} l_{2, j}+\left|C_{j}\right| \geq\left(\frac{1}{2 \sigma-2}-\varepsilon\right)\left(\lambda_{j}+1\right) l_{2, j},
$$

and so,

$$
\left|C_{j}\right| \geq l_{2, j}\left(\frac{1}{2 \sigma-2}-\varepsilon+\lambda_{j}\left(\frac{1}{2 \sigma-2}-\varepsilon-X_{j}\right)\right) .
$$

Now, we know that $\left|C_{j}\right| \leq l_{2, j}$, and therefore we obtain the upper bound

$$
\lambda_{j} \leq\left(\frac{2 \sigma-3}{2 \sigma-2}+\varepsilon\right)\left(\frac{1}{2 \sigma-2}-\varepsilon-X_{j}\right)^{-1} .
$$

It remains to recall that $X \leq \alpha$ to obtain

$$
\lambda \leq \frac{2 \sigma-3}{2 \sigma-2}\left(\frac{1}{2 \sigma-2}-\alpha\right)^{-1}
$$

Let us take as a new sequence $\left(y_{j}\right)_{j \geq 1}$ a subsequence such that $\lim _{j \rightarrow \infty} \lambda_{j}$ $=\lambda$. It suffices to look again at the relation (14) to obtain

$$
r_{j}=\frac{\left|C_{j}\right|}{l_{2, j}} \geq \frac{1}{2 \sigma-2}+\lambda\left(\frac{1}{2 \sigma-2}-X\right)
$$

for infinitely many values of $j$.

Then, a last extraction of a subsequence allows us to suppose that the bounded sequence $\left(r_{j}\right)_{j \geq 1}$ has a limit $r$ such that

$$
r \geq \frac{1}{2 \sigma-2}+\lambda\left(\frac{1}{2 \sigma-2}-\alpha\right) .
$$

Hence, putting together (11), (12), Lemma 3.7 and (15) we conclude the proof of the archimedean case of Theorem 1.2.

\section{References}

[1] Y. Bilu, Addition of sets of integers of positive density, J. Number Theory 64 (1997), 233-275.

[2] - Structure of sets with small sumset, Astérisque 258 (1999), 77-108.

[3] G. A. Freiman, Foundations of a Structural Theory of Set Addition, Translat. Math. Monographs 37, Amer. Math. Soc., 1973.

[4] H. Halberstam and K. F. Roth, Sequences, 2nd ed., Springer, New York, 1983.

[5] R. Jin, Inverse problem for upper asymptotic density, Trans. Amer. Math. Soc. 355 (2002), 57-78.

[6] - Solution to the inverse problem for upper asymptotic density, to appear.

[7] M. Kneser, Abschätzung der asymptotischen Dichte von Summenmengen, Math. Z. 58 (1953), 459-484.

[8] V. F. Lev and P. Y. Smeliansky, On addition of two distinct sets of integers, Acta Arith. 70 (1995), 85-91. 
[9] M. B. Nathanson, Additive Number Theory. Inverse Problems and the Geometry of Sumsets, Grad. Texts in Math. 165, Springer, 1996.

[10] Y. Stanchescu, On addition of two distinct sets of integers, Acta Arith. 75 (1996), 191-194.

Département de Mathématiques

Université de Bordeaux 1

Talence Cedex, France

E-mail: bordes@math.u-bordeaux1.fr

Received on 30.7.2004

and in revised form on 7.6.2005 